\title{
Interfaces modify the undulation spectrum of bicontinuous microemulsions
}

\author{
O. Holderer ${ }^{1, \text { a }}$, F. Lipfert ${ }^{1}$, H. Frielinghaus ${ }^{1}$, M. Ohl ${ }^{2}$ and D. Richter ${ }^{3}$ \\ ${ }^{1}$ Jülich Centre for Neutron Science, Forschungszentrum Jülich GmbH, Lichtenbergstrasse 1, 85747 Garching, Germany \\ ${ }^{2}$ Jülich Centre for Neutron Science, Outstation at SNS, Forschungszentrum Jülich GmbH, Oak Ridge, USA \\ ${ }^{3}$ Institue for Complex Systems 1: Neutron Scattering, Forschungszentrum Jülich GmbH, 52425 Jülich, Germany
}

\begin{abstract}
Recently, the structure and dynamics of microemulsions adjacent to a planar hydrophilic wall have been characterized using grazing incidence small angle neutron scattering (GISANS) and grazing incidence neutron spin echo (GINSES) spectroscopy, respectively. It has been found that a bicontinuous microemulsion develops a lamellar structure, and that the relaxation rate is faster for a membrane close to the interface compared to the bulk. A model developed by Seifert was employed to explain the discovered acceleration for the near-surface lamellar ordered membranes. In this contribution we present how confinement of a flat interface influences the elastic properties of surfactant membranes and discuss the effect of locally introduced flat interfaces by clay platelets. This analysis sheds light onto the undulation mode spectrum of the surfactant membrane.
\end{abstract}

\section{Introduction}

Quasielastic neutron scattering is a well-suited technique for studying thermal fluctuations in soft matter systems. We study the interfacial membrane in bicontinuous microemulsions, a thermodynamically stable mixture of oil, water and a surfactant. A monolayer of the surfactant separates the water and oil domains. The average distance between surfactant layers (of the extent of a water or oil domain) is of the order of $10 \mathrm{~nm}$. The structure of such systems is widely studied with small angle neutron scattering (SANS) [1,2], while the membrane undulations of the surfactant membranes lay in the energy range accessible by quasielastic neutron scattering techniques such as neutron spin echo (NSE) spectroscopy [3]. Recently, the influence of a rigid interface on the structural and elastic properties of surfactant layers has been investigated with neutron scattering techniques. It has been found experimentally that the structure of bicontinuous microemulsions changes near an interface and forms some layers of a lamellar phase [4]. This is in agreement with computer simulations. Figure 1 illustrates the situation in the vicinity of a rigid interface.

\section{Methods}

Neutron scattering techniques have been used to investigate the structure and dynamics of microemulsions. Experiments have been carried out at the MLZ in Garching at the FRM II research reactor and at the Spallation Neutron Source (SNS) of the Oak Ridge National Lab.

\footnotetext{
${ }^{a}$ Corresponding author: o.holderer@fz-juelich.de
}

\subsection{Structure}

\subsubsection{SANS}

Small angle neutron scattering provides structural information on the mesoscopic length scale in reciprocal space. The magnitude of the scattering vector $\mathrm{q}$ is hereby related to the real space distance $\mathrm{d}$ by $\mathrm{q}=2 \pi / \mathrm{d}$.

\subsubsection{GISANS}

If sensitivity to surface structures is required, grazing incidence SANS (GISANS) is the method of choice. Similar to a typical neutron reflectometry experiment of liquid samples, the microemulsion is kept behind a silicon block with the neutron beam hitting the interface between silicon and sample. By choosing an angle below the critical angle of reflection, an evanescent neutron wave penetrates the sample behind the silicon block. The scattering depth can be varied by varying the incident angle and/or varying the contrast between silicon block and sample (by choosing the right ratio of deuterated and protonated components in the sample).

\subsection{Dynamics}

\subsubsection{NSE}

The length- and timescale of NSE spectroscopy matches the local processes in bicontinuous microemulsions perfectly. In this context, the same length scales as covered with SANS are probed with NSE. By scanning the Fourier time at a given scattering vector $\mathrm{q}$, the intermediate scattering function $\mathrm{S}(\mathrm{q}, \mathrm{t})$ is measured, which is the Fourier transform of the scattering function $S(q, \omega)$ measured e.g. with Time-of-Flight spectroscopy or backscattering spectroscopy. On the other hand it is the Fourier

This is an Open Access article distributed under the terms of the Creative Commons Attribution License 4.0, which permits unrestricted use, distribution, and reproduction in any medium, provided the original work is properly cited. 


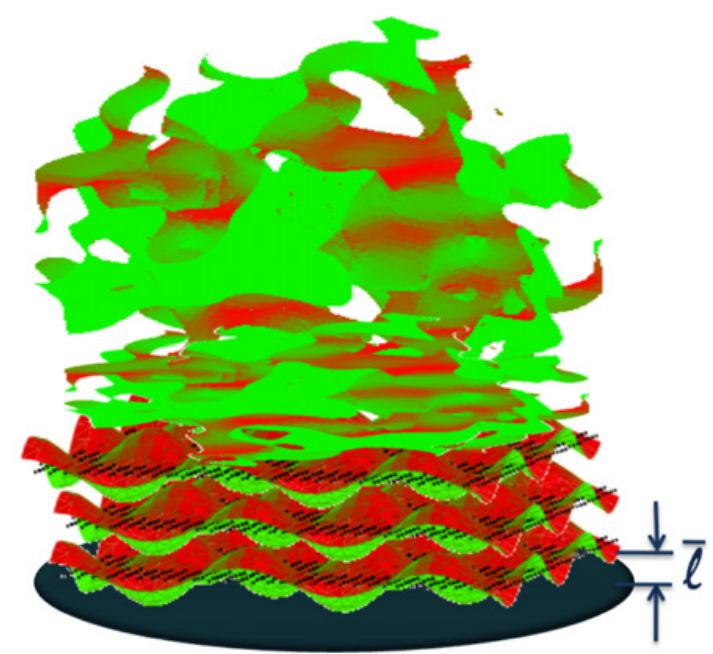

Figure 1. Schematic representation of a bicontinuous microemulsion in the vicinity of a rigid wall. The distance $\bar{\ell}$ determines how much the microemulsion is affected by the wall.

transform of the real space correlation function $G(r, t)$. NSE experiments have been carried out at the J-NSE spectrometer at the MLZ [5,6] and at the SNS-NSE spectrometer in Oak Ridge [7,8].

\subsubsection{GINSES}

The same geometry as used in GISANS experiments can also be applied at the NSE spectrometer. If only an evanescent wave of a laterally collimated beam hits the sample, the intensity penalty is huge (of the order of $1 / 10^{5}$ ). Microemulsions are in this sense a very favourable system with rather large scattering intensity. All GINSES measurements were performed so far at a relatively small qvalue of $0.08 \AA^{-1}$ as a compromise between high intensity (at lower q) and probing the local dynamics (at higher q).

Figure 2 shows the echo group of one of the first GINSES experiments (top) and a later one after optimization (bottom). The dashed lines indicate the "spin up" configuration (all flippers off, maximum intensity at the given setup) and "spin down" configuration (minimum intensity, only $\pi$-flipper on). The count rate at the "spin down" configuration during the first experiment indicates that half of the detected neutrons are depolarized background (general background in the neutron guide hall, remaining depolarized background of the J-NSE spectrometer itself). A very stable instrument without thermal drifts of the power supplies or changes of the magnetic field due to temperature changes of the coils as well as a low and stable background of the other instruments is needed for such experiments. It was possible to accept count rates of 3-4 cps ("spin up - spin down") over a background of $3 \mathrm{cps}$. Optimization mainly concerning the sample cell lead to count rates of $\sim 25 \mathrm{cps}$ for the microemulsion experiments. An important point in this respect is the flatness of the Si-block. A lower roughness increased the intensity of the detected scattered neutrons. The second path of optimization was to increase the hight of the cell and in this way the sample area in the beam.

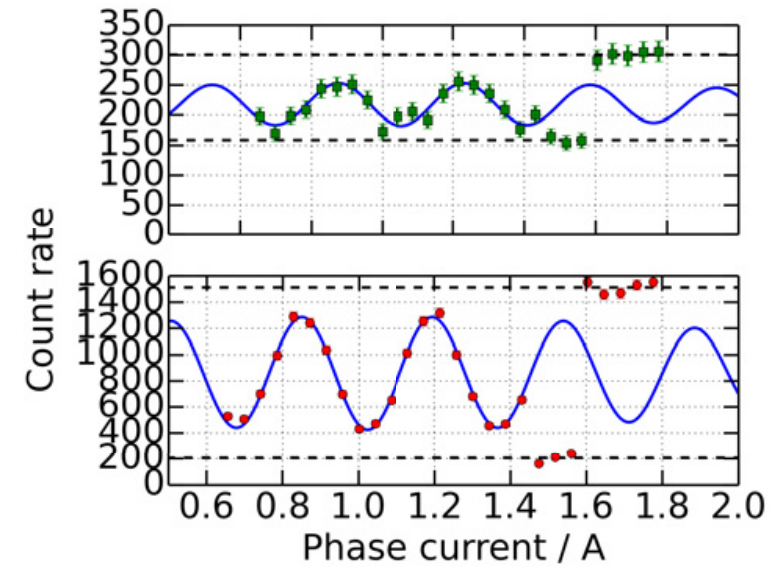

Figure 2. Neutron spin echo group at $5 \mathrm{~ns}$ under grazing incidence conditions measured at J-NSE. Top: first experiments with 50\% background; bottom: optimized experiment. The dashed lines indicate the spin up and spin down configuration, i.e. the maximum and minimum count rate for a given setting.

\section{Results and discussion}

The focus was set on investigating the influence of rigid interfaces to the relaxation rate of surfactant membranes in microemulsions. The relaxation rate allows to deduce the bending rigidity of the membrane. Bicontinuous microemulsions used here consisted of equal amounts of water and oil (decane), and $17 \mathrm{vol} \%$ of the non-ionic surfactant $\mathrm{C}_{10} \mathrm{E}_{4}$. The standard composition consisted of $\mathrm{D}_{2} \mathrm{O}$, protonated decane and protonated surfactant ("bulk contrast" between water domains and oil+surfactant domains). Variation of the H2O/D2O mixture allowed to change the contrast in the GINSES experiments and to vary the penetration depth (besides varying the incident angle). The membranes close to the interface, which form the lamellar phase, show a faster relaxation than the membranes in the bulk microemulsion. This has been interpreted as a modification of the undulation mode spectrum affecting mainly the long wavelength modes of the membrane $[9,10]$. The Helfrich Hamiltonian describing the free energy of a surfactant membrane is modified by the presence of a solid interface as described by the theory of Seifert [11] due to the wall-membrane interaction. The dispersion relation of membrane undulations change from a $\mathrm{k}^{3}$ to $\mathrm{k}^{2}$ behaviour (with the undulation wave vector $\mathrm{k}$ ), the influence of the wall is described by the distance of the first membrane to the wall, $\bar{\ell}$ (see Fig. 1 for the definition of $\bar{\ell}$ ).

In bulk microemulsions, the relaxation rate measured with NSE depends on the structure (the relevant values are typically determined in SANS experiments) and the bending rigidity of the membrane. NSE allows therefore to determine the bending rigidity $\kappa$ of surfactant membranes. At the interface, the quantity $\bar{\ell}$ is also influencing the relaxation rate. Figure 3 shows the interplay between $\kappa$ and $\bar{\ell}$. Stiffer membranes and larger distances of the first layer imply an increase of the relaxation time. For the formula of the intermediate scattering function of membranes with the Seifert formalism we refer to references [9] and [10]. 


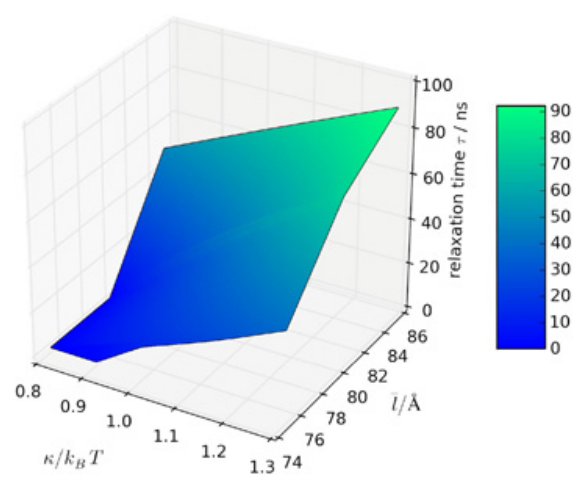

Figure 3. Dependency of the relaxation rate on the bending rigidity and the distance of the first membrane to the rigid wall.

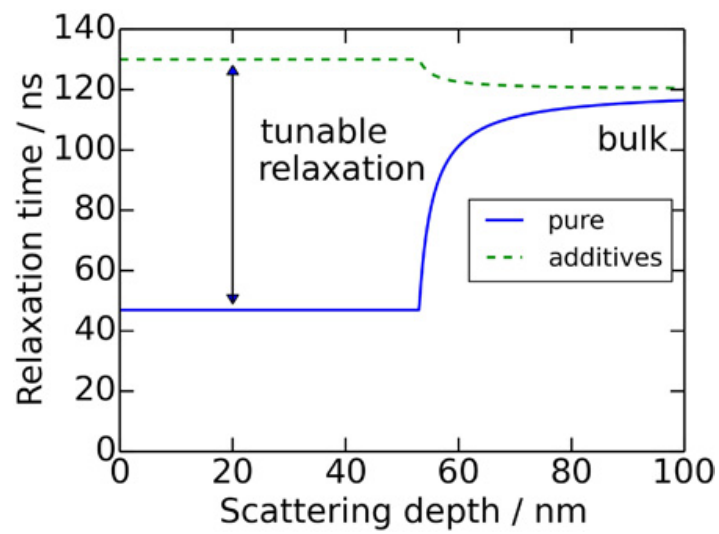

Figure 4. Behaviour of the relaxation time of a surfactant membrane close to an interface. Additive addition allows tuning the elastic properties at the interface.

It has turned out that the relaxation time can be modified by the addition of diblock copolymers, which are used as "boosters" in bicontinuous microemulsion, i.e. a small amount of them improves the efficiency of the surfactant and allows to emulsify much more water with oil at a given surfactant concentration [10]. GISANS experiments showed that the diblock copolymers accumulate at the first layers close to the rigid interface. The relaxation time also increases compared to the relaxation time at the interface of a pure microemulsion. Figure 4 shows schematically the region over which the interface relaxation time can be varied in this kind of microemulsions. The change in relaxation rate has been interpreted as a change of the spectrum of undulation modes, with an increase of the amplitude of long wavelength fluctuations and a decrease of the frequencies of the near-interface undulations due to the polymer addition.

Locally, flat interfaces may be introduced into a microemulsion by the addition of clay particles, which are basically flat discs. The dynamics of bicontinuous microemulsions with added small clay particles (Laponite, diameter $\sim 80 \mathrm{~nm}$ ) showed no change of the dynamics [12]. Recently, the same experiment has been repeated with much larger clay particles (Nanofil, diameter $>200 \mathrm{~nm}$ ). Figure 5 shows the intermediate scattering function of a pure microemulsion and a microemulsion with large clay

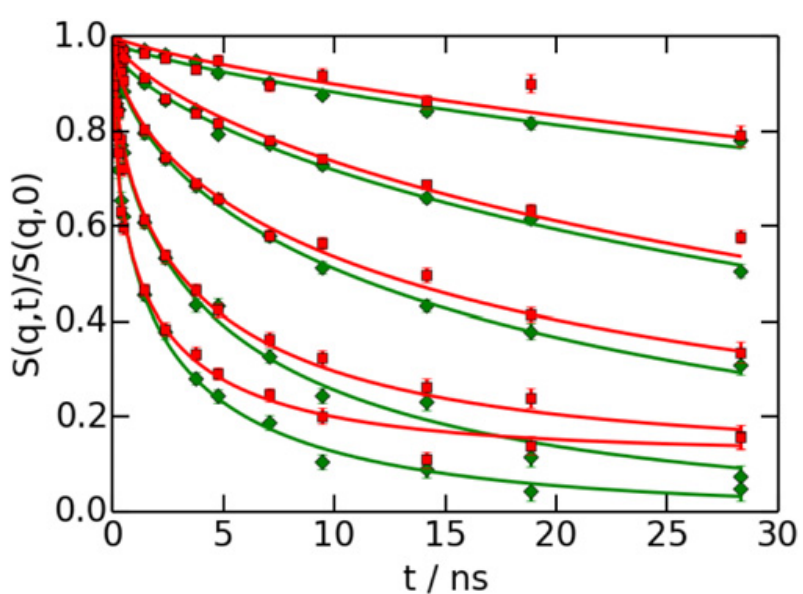

Figure 5. Intermediate scattering function measured with the SNS-NSE. Green diamonds: pure microemulsion; Red squares: microemulsion with large clay particles. From top to bottom the q-values have been $0.05,0.08,0.11,0.15$ and $0.18 \AA^{-1}$.

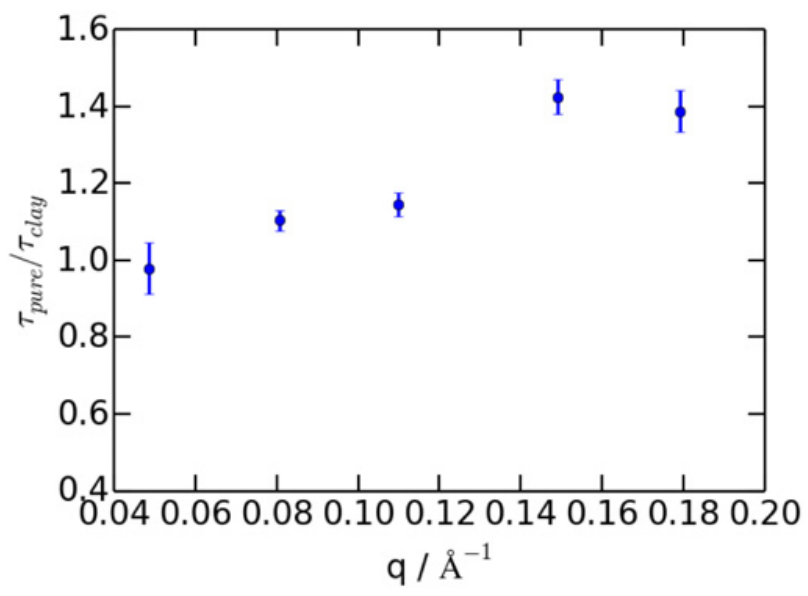

Figure 6. Ratio of relaxation times of the pure microemulsion and the one with added large clay particles as a function of $\mathrm{q}$, showing that the relaxation gets faster with the clay, the effect being more pronounced for smaller length scales (large q).

particles. It was attempted to match the average scattering length density of the oil/water mixture of the microemulsion in film contrast, where only the $\mathrm{C}_{10} \mathrm{E}_{4}$-surfactant layer remains visible against the rest of the components h-decane $(33 \%) / d$-decane $(67 \%), \mathrm{H} 2 \mathrm{O}(30 \%) / \mathrm{D} 2 \mathrm{O}(70 \%)$, as it has been made successfully with the laponite particles [12]. The more natural clay resulted in a less well matched system as for the Laponite case, as can be seen in the remaining plateau value at the highest q-value $0.18 \AA^{-1}$ (red squares). This has been confirmed also in SANS experiments. The value of the elastic background has been taken from SANS experiments as an additional elastic contribution, it was no free parameter during the fitting of the data (diffusion of the large clay particles plays no significant role in the NSE time window). The relaxation time has been determined from a fit with a stretched exponential function, which is often used in the analysis of the dynamics of bicontinuous microemulsions (see e.g. the procedures in Ref. 3) plus the aforementioned constant 
elastic contribution. It turned out that the relaxation time is indeed decreased with the large clay particles. Figure 6 shows this trend as a function of $\mathrm{q}$. This is reasonable when considering the typical length scale of the transition from $\mathrm{k}^{3}$ to $\mathrm{k}^{2}$ dependence of the dispersion relation to be $\mathrm{d}=$ $2 \pi / \mathrm{q} \approx 50 \mathrm{~nm}[9,10]$, i.e. the long wavelength undulations affected by the interface can not develop in the Laponite sample because the clay particles are simply too small, while they are already present with the larger Nanofil clay particles added to the microemulsion.

\section{Conclusions}

Neutron scattering has been well suited for studying the elastic properties of bicontinuous microemulsions and the effects of confinement imposed to the surfactant membrane by a rigid interface. Neutron spin echo spectroscopy probes the right length- and time-scale for such investigations. The recently developed method of grazing incidence NSE has been proven to be very useful for studying the dynamics of surfactant membranes close to a rigid interface. Besides, also other aspects of soft matter at interfaces, e.g. the influence of hydrophilic or hydrophobic interfaces on micelles [13] can be studied with GINSES. An increase in intensity could open many new possible experiments in this interface relates soft matter topic. In pure microemulsions, GINSES experiments showed that the relaxation time gets faster close to the interface due to the membrane-wall interactions. The behaviour can be modified and tuned by the addition of diblock copolymers, which accumulate at the interface and change the undulation mode spectrum. Introducing clay interfaces into the microemulsion confirmed this concept of a changed undulation mode spectrum, only clay particles with a sufficient diameter such that long wavelength undulations can develop show a modified microemulsion dynamics. We think that these findings have implications in the rheology and tribology in systems containing microemulsions and interfaces, e.g. in cosmetics applications.

This work is based on experiments performed at the Heinz Maier-Leibnitz Zentrum (MLZ), Garching, Germany, and the SNS-NSE at ORNL, Oak Ridge, USA. Part of the research conducted at ORNL's Spallation Neutron Source was sponsored by the Scientific User Facilities Division, Office of Basic Energy Sciences, US Department of Energy.

\section{References}

[1] M. Teubner, R. Strey, J. Chem. Phys., 87, 3195 (1987)

[2] D. Byelov, H. Frielinghaus, O. Holderer, J. Allgaier, D. Richter, Langmuir, 20, 10433 (2004)

[3] M. Mihailescu, M. Monkenbusch, H. Endo, et al., J. Chem. Phys. 115, 9563 (2001)

[4] M. Kerscher, P. Busch, S. Mattauch, H. Frielinghaus, D. Richter, M. Belushkin, G. Gompper, Phys. Rev. E, 83, 030401 (2011)

[5] O. Holderer, M. Monkenbusch, G. Borchert, C. Breunig, and K. Zeitelhack, Nucl. Instr. Meth. Phys. Res. Sec. A 586, 90 (2008)

[6] O. Holderer, M. Monkenbusch, R. Schätzler, H. Kleines, W. Westerhausen, and D. Richter, Measurement science and technology, 19, 034022 (2008)

[7] M. Ohl, M. Monkenbusch, N. Arend, T. Kozielewski, G. Vehres, C. Tiemann, M. Butzek, H. Soltner, U. Giesen, R. Achten, H. Stelzer, B. Lindenau, A. Budwig, H. Kleines, M. Drochner, P. Kaemmerling, M. Wagener, R. Moeller, E.B. Iverson, M. Sharp, D. Richter, Nucl. Instr. Meth. Phys. Res. Sec. A 696, 85 (2012)

[8] M. Ohl, M. Monkenbusch, D. Richter, C. Pappas, K. Lieutenant, T. Krist, G. Zsigmand, F. Mezei, Physica B 350, 147 (2004)

[9] H. Frielinghaus, M. Kerscher, O. Holderer, M. Monkenbusch, D. Richter, Phys. Rev. E, E85, 041408 (2012)

[10] F. Lipfert, H. Frielinghaus, O. Holderer, S. Mattauch, M. Monkenbusch, N. Arend, D. Richter, Phys. Rev. E, 89, 042303 (2014)

[11] U. Seifert, Phys. Rev E, 49, 3124 (1994)

[12] H. Frielinghaus, O. Holderer, F. Lipfert, M. Kerscher, S. Mattauch, D. Richter, Eur. Phys. J. Web of Conf., 33, 03005 (2012)

[13] M. Walz, S. Gerth, P. Falus, M. Klimczak, T.H. Metzger, A. Magerl, J. Phys.: Condens. Mat. 23, 324102 (2011) 